\title{
Source flash dans un gaz rare sous pression amorcée par plasma laser
}

\author{
P. Laporte, M. Bon et M. Hemici
}

Laboratoire de Traitement du Signal et Instrumentation CNRS-URA 842, Université Jean Monnet, 23 rue du docteur Paul Michelon, 42023 Saint-Etienne Cedex 2, France

\begin{abstract}
:
Preliminary results on a plasma laser triggered spark source in a rare gas are reported. Simple and stable geometry of the spark was achieved using a coaxial device including a hollow cathode for the laser beam focusing. Time width of typically $200 \mathrm{~ns}$ in the UV are obtained together with almost jitter free operation.
\end{abstract}

Dans un certain nombre d'études où interviennent des phénomènes transitoires il est nécessaire de disposer de sources de haut flux, brèves et synchronisables avec précision. C'est dans cette perspective qu'une source VUV résultant d'un claquage laser dans un gaz rare a été étudiée précédemment au laboratoire (1). Dans la présente contribution nous donnons les résultats préliminaires obtenus en couplant le principe du plasma laser et de la décharge haute tension dans un gaz rare sous pression. Le plasma laser permet alors le déclenchement de la décharge électrique.

L'objectif est l'obtention de flux par impulsion plus élevés tout en conservant le caractère synchronisable de la source. Le principe du déclenchement d'une décharge par plasma laser a également été récemment étudié (2) dans le cas d'une source à étincelle sous vide, donnant une source dérivée de la source dite " B.R.V." (3). La capacité de cette source à être synchronisée a été démontrée dans des expériences d'excitation séquentielle (4). Les essais effectués à l'aide du prototype réalisé n'incluent pas encore le couplage à un monochromateur sous vide, mais permettent déjà de dégager certaines conclusions intéressantes.

Un premier montage dans lequel le plasma laser était réalisé par focalisation transversale par rapport aux électrodes conduisait à une décharge de géométrie complexe empruntant la colonne de plasma transversale (fig. 1a). Le système décrit ici adopte une géométrie originale où le plasma est formé longitudinalement en mettant en oeuvre une "cathode creuse" (fig. 1b).

Ce dispositif permet d'obtenir une décharge bien confinée et stable, ce qui la rend $a$ priori favorable pour un couplage avec la fente d'entrée d'un monochromateur sous vide. Pour des raisons de commodité, la lumière a été recueillie à l'aide d'une fibre optique couplée à un monochromateur visible. L'analyse spectrale est obtenue à l'aide d'un détecteur type OMA, et l'analyse temporelle avec une photodiode rapide couplée à un oscilloscope à mémoire numérique rapide ( $1 \mathrm{G}$ éch./sec) permettant l'étude détaillée des monocoups.

La liaison capacité-cellule possède une géométrie réduisant au maximum l'inductance. 


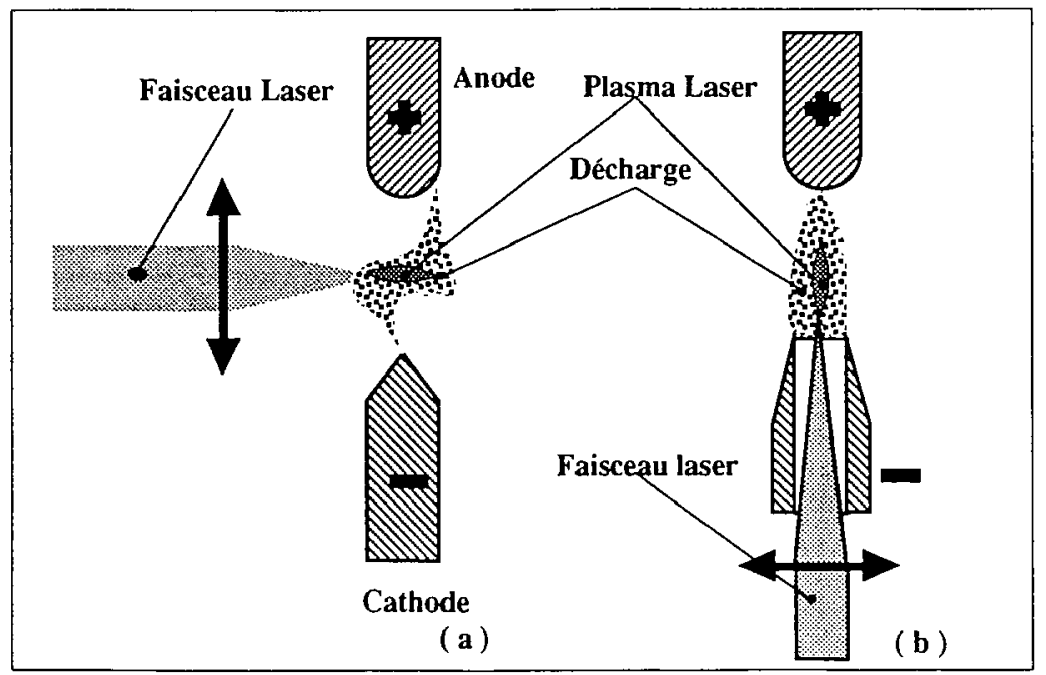

Fig. 1: Principe de la source: (a) amorçage transversal; (b) amorçage longitudinal.

Les conditions typiques d'expériences sont :

-Capacité : $100 \mathrm{nF}$ - Haute tension $5-10 \mathrm{kV}$ - Cadence $5-10 \mathrm{~Hz}$ -

- Pression de gaz 0,5 - 10 bar - Energie laser $\approx 100 \mathrm{~mJ}(12 \mathrm{~ns}$.)

Les caractéristiques de la décharge et de l'émission sont typiquement :

- Courant : Oscillations amorties de période $\approx 500$ ns (ceinture de Rogowsky).

- Largeur temporelle d'autant plus courte que la longueur d'onde décroit (émission corps noir / thermalisation ). Les temps observés sont de l'ordre de 200 ns pour la largeur à mi-hauteur à $350 \mathrm{~nm}$ (fig. 2).

- Emission spectrale montrant de nombreuses raies atomiques et ioniques dans le visible et un pseudo-continuum dans l'UV (étude à haute résolution non représentée ici).



Fig.2 : émission en fonction du temps à différentes longueurs d'onde 
Le système provoque peu de projections, l'essentiel de la décharge ne mettant pas en jeu le métal de l'anode. L'amorçage de la source est possible jusqu'à des pressions élevées (essais à 8 bar). Le jitter est dans la gamme de la nanoseconde, sauf à haute pression où il faut augmenter l'énergie du laser d'amorçage.

Diverses améliorations du système ainsi que le couplage à un monochromateur VUV sont en cours de développement.

1- P. Laporte, N. Damany and H. Damany

Pulsed laser generated rare gas plasma as a light source in the vacuum ultraviolet.

Opt. Letters, 12, 987 (1987)

2- H. Damany and P. Laporte

Triggering of a vacuum spark source by impact of a laser beam on the cathode.

Rev. Sci. Instr. 60, 602 (1989)

3- G. Balloffet, J. Romand and B. Vodar

C.R. Acad. Sci. 252, 4139 (1961)

4- P. Moutard, Thèse, Lyon (1986) 